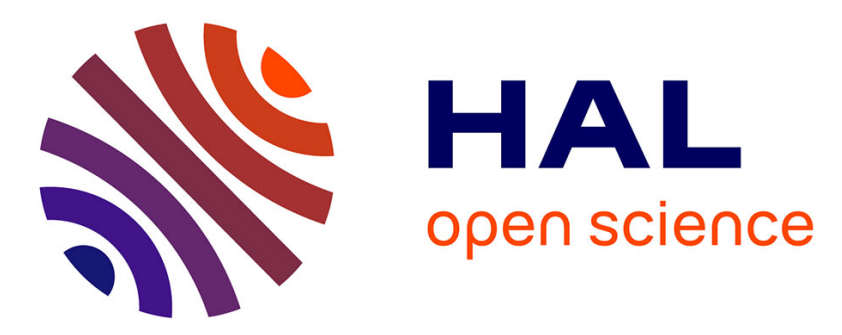

\title{
Efficient borylation of reactive aryl halides with MPBH (4,4,6-trimethyl-1,3,2-dioxaborinane).
}

Nageswaran Praveenganesh, Emilien Demory, Christine Gamon, Véronique Blandin, Pierre Yves Chavant

\section{- To cite this version:}

Nageswaran Praveenganesh, Emilien Demory, Christine Gamon, Véronique Blandin, Pierre Yves Chavant. Efficient borylation of reactive aryl halides with MPBH (4,4,6-trimethyl-1,3,2-dioxaborinane).. SYNLETT, 2010, 2010 (16), pp.2403-2406. 10.1055/s-0030-1258059 . hal-00520331

\section{HAL Id: hal-00520331 https://hal.science/hal-00520331}

Submitted on 22 Sep 2011

HAL is a multi-disciplinary open access archive for the deposit and dissemination of scientific research documents, whether they are published or not. The documents may come from teaching and research institutions in France or abroad, or from public or private research centers.
L'archive ouverte pluridisciplinaire HAL, est destinée au dépôt et à la diffusion de documents scientifiques de niveau recherche, publiés ou non, émanant des établissements d'enseignement et de recherche français ou étrangers, des laboratoires publics ou privés. 


\title{
Efficient borylation of reactive aryl halides with MPBH (4,4,6-trimethyl- 1,3,2-dioxaborinane).
}

\author{
France \\ Fax: (+)33 476635983. Pierre-Yves.Chavant@ujf-grenoble.fr \\ PUBLISHED in \\ SYNLETT 2010, No.16, pp 2403-2406 DOI: 10.1055/s-0030-1258059 \\ (C) Georg Thieme Verlag Stuttgart, New York
}

Nageswaran PraveenGanesh, Emilien Demory, Christine Gamon, Véronique Blandin, Pierre Y. Chavant*

Département de Chimie Moléculaire, UMR-5250, ICMG FR-2607, CNRS, Universite Joseph Fourier, BP 53, 38041 Grenoble Cedex 09,

\begin{abstract}
The combination of 4,4,6-trimethyl-1,3,2dioxaborinane, a particularly stable and inexpensive borylation reagent, and Buchwald's palladium catalyst provides a simple, fast, cost-effective borylation of electron-rich, reactive iodides, bromides, and triflates, to produce stable, easily purified boronic esters.
\end{abstract}

Key words: boron, palladium, catalysis, arylboronic, Suzuki

The increasing number of applications of the SuzukiMiyaura coupling prompts the design of efficient and cost-effective methods for the preparation of the arylboronic precursors. Among these, the borylation of aryl halides or triflates with pinacolborane ${ }^{1}(\mathrm{PinBH})$ is an excellent alternate to the more classical addition of organometallics to trialkoxyborane. Murata's team ${ }^{2}$ and ours $^{3}$ proposed the replacement of $\mathrm{PinBH}$ in this reaction by the easily accessible, much less expensive and more conveniently prepared MPBH (4,4,6-trimethyl-1,3,2dioxaborinane $\left.^{4} \mathbf{1}\right)$. In addition, Billingsley and Buchwald $^{1 \mathrm{i}}$ completed in 2008 the original study of Baudoin et $a .^{1 \mathrm{c}}$ on the very positive role of bulky phosphine ligands in the borylation with $\mathrm{PinBH}$. We wondered if the advantages of both implementations could be combined to provide a convenient and cost-effective access to aryl boronic esters from aryl halides; we discuss herein the advantages and limitations of this system.



Scheme 1 Preparation of MPBH 1. Reagents and conditions: dioxane, $20^{\circ} \mathrm{C}, 30 \mathrm{~min}$.

MPBH is a particularly stable dioxaborinane, ${ }^{4}$ readily accessible from hexyleneglycol and borane. We described earlier ${ }^{2,3}$ convenient preparations using $\mathrm{BH}_{3}-$ DMS or $\mathrm{B}_{2} \mathrm{H}_{6}$ generated from $\mathrm{NaBH}_{4}$ and $\mathrm{MeSO}_{3} \mathrm{H}$. We considered the use of $N, N$-diethylaniline-borane, a safe ${ }^{5}$ source of borane for large-scale applications, but the reaction of hexylene glycol with this reagent was sluggish. $\mathrm{Pd} / \mathrm{C}$ is known ${ }^{6}$ to accelerate the decomplexation and solvolysis of amine-boranes in methanol. In an analogous manner (Scheme 1), we found that reacting $N, N$-diethylaniline-borane and hexyleneglycol in the presence of $\mathrm{Pd} / \mathrm{C}$ ( $5 \%$ by weight of $10 \% \mathrm{Pd} / \mathrm{C}$ reagent) resulted in the complete consumption of the diol in 30 min at room temperature. Distillation of the reaction mixture in vacuo provided neat MPBH in $75 \%$ yield. ${ }^{7,8}$

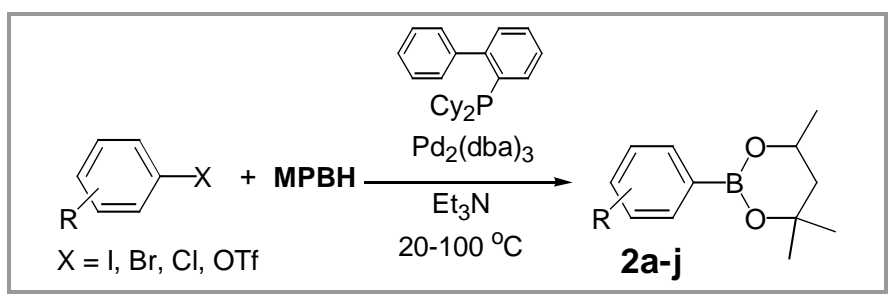

Scheme 2 Borylation of aryl halides and triflates.

Then we studied the palladium-catalyzed borylation of various aryl halides and triflates with $\mathrm{MPBH}$ and a catalyst prepared from $\mathrm{Pd}_{2}(\mathrm{dba})_{3}$ and CyJohnPhos ${ }^{9}$ according to Baudoin's procedure ${ }^{1 \mathrm{c}}$ (Scheme 2, Table 1). ${ }^{10}$ In preliminary experiments, we found that toluene and dioxane were equally suitable solvents.

With PinBH, a dramatic feature of this catalyst system compared to others ${ }^{1-3}$ is the extreme rapidity of the process, when applied to electron-rich aryl iodides ${ }^{11}$ or bromides. $^{12}$ The same is true with MPBH. This high reactivity allows complete conversion of reactive aryl iodides and bromides (entries $1-4,7,8$ ) between 20 and $40^{\circ} \mathrm{C}$ within a few hours. To our knowledge, operating at $20^{\circ} \mathrm{C}$ has not been described for this reaction yet. ${ }^{13}$

The process remained efficient at low levels of catalyst: the boronate ester was produced from $p$-bromo- $N, N$ dimethylaniline in $90 \%$ yield in $6 \mathrm{~h}$ at $20^{\circ} \mathrm{C}$ when 0.3 mol\% Pd was used (10 mmol run, entry 7).

When less reactive iodides were used, some hydrodehalogenation was observed. This side-reaction is always met in analogous borylation with $\mathrm{PinBH}$, and is favored by electro-withdrawing substituents on the aryl ring. In the present work as well as in the literature, it is the only side-reaction: the homocoupling product was never observed. We indicate whenever available the Ar$\mathrm{B}(\mathrm{OR})_{2} / \mathrm{Ar}-\mathrm{H}$ ratio, but low-boiling arenes were seldom accurately measurable. Note that all reactions of Table 1 were run to complete conversion of the starting material. Thus, lower isolated yields reflect a higher level of hydrodehalogenation. With the reactive substrates of entries $1-4,7$ and 8 , the reduced product was below detec- 
tion. In all cases, it was found to be less present than with the $\mathrm{PdCl}_{2}$ (TPP $)_{2}$ catalyst in our former work. ${ }^{3}$ It can be concluded that in the case of reactive, electron-rich aryl iodides and bromides, MPBH is a cost-effective substitute to pinacolborane in a fast and efficient palladium-catalyzed borylation.

Disappointingly, with less reactive aryl halides, a clearcut difference in reactivity between PinBH and MPBH appeared, with MPBH favouring the unwanted hydrodehalogenation. The difference remained limited with electron-depleted iodides (entries 5 and 6). Entry 5 (69\% yield) should be compared with Masuda's ${ }^{1 b}$ result: borylation of ethyl 4-iodobenzoate with PinBH in the presence of $\mathrm{PdCl}_{2} \mathrm{dppf}$ yielded $79 \% \mathrm{Ar}$-Pin (hydrodehalogenated product $17 \%$ ). More dramatic differences appeared with electron-depleted bromides, for which hydrodehalogenation became deleterious (entries 9-12). In the same conditions, PinBH was much more efficient: compare entries 9 and 10,11 and 12 .

The borylation of chlorides was practically limited to the most reactive, electron-rich ones (entries 15-17). ${ }^{14}$ Remarkably, aryl triflates ${ }^{1 \mathrm{~b}, 15}$ were borylated efficiently (entries 13-14). The extent of the hydrodetriflation seemed less dependent of the electro-donating (entry 13) or electro-withdrawing nature of the substituents (entry 14, compare also with entry 11).

So far, all reported optimizations of this reaction were done with electron-rich substrates. ${ }^{1-3}$ We decided to check the influence of reaction parameters on the borylation-hydrodehalogenation ratio when the substrate is electron-depleted; 2-bromonaphtalene was our model of "unreactive" substrate. ${ }^{16}$ Reactions were monitored by GC with internal standard. Although we could not increase the yield in boronic ester $\mathbf{2 g}$ above $50 \%$, some interesting trends appeared. Higher temperatures favor borylation over hydrodehalogenation (37\% yield in $\mathbf{2 g}$ at $80^{\circ} \mathrm{C}, 50 \%$ at $150^{\circ} \mathrm{C}$ ), making this reaction a good candidate for closed-vessel, microwaves-heated reactions. Nevertheless, at identical temperatures, microwaves heating did not lead to significantly faster reaction than conventional heating. With 2-bromonaphtalene and MPBH, other ligand systems (dppf, ${ }^{1 \mathrm{a}, \mathrm{b}}$ dpePhos, ${ }^{1 \mathrm{f}}$ excess $\mathrm{TPP}^{3}{ }^{N} N$-(dicyclohexylphosphino)-2-(2'-tolyl)indole ${ }^{17}$ or di-tert-butylbipyridyl) led to slower reactions and increased hydrodehalogenation.

Monitoring the course of the reaction showed that the borylation/hydrodehalogenation ratio is lower at early stages of the reaction. Examples have been reported with $\mathrm{PinBH}^{18}$ where prolonged heating of the reaction mixtures caused an increase of the hydrodehalogenation. In the present conditions, both reactions are concurrent. Prolonged heating after the end of the conversion of the bromide never caused any significant loss of borylated product. A larger excess of MPBH (3 eq.) slightly favored hydrodehalogenation: best results were obtained with only 1.5 eq. dialkoxyborane.

Our hypothesis is that MPBH is more prone than PinBH to some disproportionation ${ }^{19}$ in the reaction conditions, releasing small amounts of $\mathrm{BH}_{3}$ that would favor Pd- catalyzed reduction of the substrate. Indeed, replacing MPBH by $\mathrm{BH}_{3}: \mathrm{Et}_{3} \mathrm{~N}$ caused extensive hydrodehalogenation.

Table 1 Borylation of various aryl halides and triflates with MPBH catalyzed by Pd/CyJohnPhos. ${ }^{\text {a,10 }}$

\begin{tabular}{|c|c|c|c|c|}
\hline Entry & substrate & conditions & $\begin{array}{l}\mathrm{ArB}(\mathrm{O} \\
\mathrm{R})_{2} / \mathrm{Ar} \\
\mathrm{H}^{\mathrm{b}}\end{array}$ & $\begin{array}{l}\text { Product } \\
\text { yield } \\
(\%)^{c}\end{array}$ \\
\hline 1 & $\mathrm{Me}_{2}$ & $\begin{array}{l}3 \% \mathrm{Pd}, 20^{\circ} \mathrm{C}, \\
<0.5 \mathrm{~h}\end{array}$ & $>99: 1$ & $\begin{array}{l}\mathbf{2 a} \\
90\end{array}$ \\
\hline 2 & $\mathrm{Me}$ & $\begin{array}{l}1 \% \mathrm{Pd}, 20^{\circ} \mathrm{C}, \\
1 \mathrm{~h}\end{array}$ & $>95: 5$ & $\begin{array}{l}\mathbf{2 b} \\
91\end{array}$ \\
\hline 3 & & $\begin{array}{l}0.5 \% \mathrm{Pd}, \\
20^{\circ} \mathrm{C}, \\
0.5 \mathrm{~h}\end{array}$ & $>97: 3$ & $\begin{array}{l}\mathbf{2 c} \\
79\end{array}$ \\
\hline 4 & & $\begin{array}{l}1 \% \mathrm{Pd}, 20^{\circ} \mathrm{C}, \\
3 \mathrm{~h}\end{array}$ & $>97: 3$ & $\begin{array}{l}\mathbf{2 d} \\
88\end{array}$ \\
\hline 5 & $\mathrm{Mec}$ & $\begin{array}{l}1 \% \mathrm{Pd}, 80^{\circ} \mathrm{C}, \\
3 \mathrm{~h}\end{array}$ & $70: 30$ & $\begin{array}{l}\mathbf{2 e} \\
69\end{array}$ \\
\hline 6 & & $\begin{array}{l}1 \% \mathrm{Pd}, 40^{\circ} \mathrm{C}, \\
16 \mathrm{~h}\end{array}$ & n.d. ${ }^{\mathrm{d}}$ & $\begin{array}{l}\mathbf{2 f} \\
60\end{array}$ \\
\hline 7 & $\mathrm{Me}$ & $\begin{array}{l}0.3 \% \mathrm{Pd}, \\
20^{\circ} \mathrm{C}, \\
6 \mathrm{~h}\end{array}$ & $>99: 1$ & $\begin{array}{l}\mathbf{2 a} \\
90^{\mathrm{e}}\end{array}$ \\
\hline 8 & & $\begin{array}{l}0.5 \% \mathrm{Pd}, \\
40^{\circ} \mathrm{C}, \\
6 \mathrm{~h}\end{array}$ & $>95: 5$ & $\begin{array}{l}\mathbf{2 b} \\
81\end{array}$ \\
\hline 10 & & $\begin{array}{l}\text { MPBH (3 eq.) } \\
1 \% \text { Pd, } 100^{\circ} \mathrm{C} \\
\text { Tol. }{ }^{\mathrm{f}}, 30 \mathrm{~h} \\
\text { PinBH }(3 \text { eq. }) \\
1 \% \text { Pd, } 100^{\circ} \mathrm{C} \\
\text { Tol. }{ }^{\mathrm{f}}, 15 \mathrm{~h}\end{array}$ & $\begin{array}{l}30: 70 \\
92: 8\end{array}$ & $\begin{array}{l}2 \mathrm{~g} \\
- \\
3 \mathbf{g} \\
-\end{array}$ \\
\hline 11 & & $\begin{array}{l}\text { MPBH } \\
1 \% \text { Pd, } 150^{\circ} \mathrm{C} \\
\text { Tol. }{ }^{\mathrm{f}}, 15 \mathrm{~min}^{\mathrm{g}} \\
\text { PinBH } \\
1 \% \text { Pd, } 150^{\circ} \mathrm{C} \\
\text { Tol. }{ }^{\mathrm{f}}, 15 \mathrm{~min}^{\mathrm{g}}\end{array}$ & $\begin{array}{l}29: 71 \\
70: 30\end{array}$ & $\begin{array}{l}\text { 2h } \\
- \\
\text { 3h } \\
\text { litt. }^{1 \mathrm{i}} 70\end{array}$ \\
\hline 13 & & $\begin{array}{l}1 \% \mathrm{Pd}, 80^{\circ} \mathrm{C}, \\
6 \mathrm{~h}\end{array}$ & n.d. & $\begin{array}{l}\mathbf{2 i} \\
82\end{array}$ \\
\hline 14 & & $\begin{array}{l}1 \% \mathrm{Pd}, 80^{\circ} \mathrm{C}, \\
16 \mathrm{~h}\end{array}$ & n.d. & $\begin{array}{l}\mathbf{2 i} \\
73\end{array}$ \\
\hline 15 & & $\begin{array}{l}1 \% \mathrm{Pd}, \\
100^{\circ} \mathrm{C}, 16 \mathrm{~h}\end{array}$ & $>95: 5$ & $\begin{array}{l}\mathbf{2 b} \\
90\end{array}$ \\
\hline 16 & & $\begin{array}{l}2 \% \mathrm{Pd}, 80^{\circ} \mathrm{C}, \\
16 \mathrm{~h}\end{array}$ & $51: 49$ & $\begin{array}{l}\mathbf{2 j} \\
45\end{array}$ \\
\hline 17 & $\mathrm{Me}$ & $\begin{array}{l}1 \% \mathrm{Pd}, 80^{\circ} \mathrm{C}, \\
24 \mathrm{~h}\end{array}$ & n.d. & $\begin{array}{l}\mathbf{2 i} \\
62\end{array}$ \\
\hline \multicolumn{5}{|c|}{$\begin{array}{l}{ }^{a} \text { Unless otherwise stated: MPBH } 1.5 \text { eq., Et }{ }_{3} \mathrm{~N} 3 \text { eq., } \\
\left.\mathrm{Pd}_{2}(\mathrm{dba})_{3}\right) \text {, CyJohnPhos } 2 \text { eq. per Pd, in dioxane. } \\
{ }^{b} \text { Measured by GC, except entries } 3 \text { and } 4:{ }^{1} \mathrm{H}-\mathrm{NMR} \text {. } \\
\mathrm{c} \text { Isolated yields after chromatography. } \\
\text { I }{ }^{\mathrm{d}} \text { n.d.: not determined. }\end{array}$} \\
\hline
\end{tabular}




\begin{tabular}{|l|}
${ }^{\mathrm{e}} 10$ mmol bromide. \\
${ }^{\mathrm{f}}$ run in toluene. \\
${ }_{\mathrm{g}}^{\mathrm{g}}$ sealed vessel, microwaves heating.
\end{tabular}

As a conclusion, the combination of MPBH, a particularly stable and inexpensive reagent, and Buchwald's palladium catalyst provides a simple, very fast, costeffective borylation of reactive, electron-rich aryl iodides, bromides, and triflates, to produce stable, easily purified boronic esters, that can be readily used as such in Suzuki couplings. 1,2

Supporting Information for this article is available online at http://www.thieme-connect.com/ejournals/toc/synlett.

\section{Acknowledgment}

We gratefully thank the Université J. Fourier and the CNRS for financial support.

\section{References}

(1) (a) Murata, M.; Watanabe, S.; Masuda, Y. J. Org. Chem. 1997, 62, 6458; (b) Murata, M.; Oyama, T.; Watanabe, S.; Masuda, Y. J. Org. Chem. 2000, 65, 164; (c) Baudoin, O. Guenard, D. Gueritte, F. J. Org. Chem. 2000, 65, 9268; (d) Melaimi, M.; Mathey, F.; Le Floch, P. J. Organomet. Chem. 2001, 640, 197; (e) Doux, M. ; Mezailles, N.; Melaimi, M.; Ricard, L.; Le Floch, P. Chem. Commun. 2002, 1566; (f) Broutin, P. E.; Cerna, I.; Campaniello, M.; Leroux, F.; Colobert, F. Org. Lett. 2004, 6, 4419; (g) Murata, M.; Sambommatsu, T.; Watanabe, S.; Masuda, Y. Synlett 2006, 1867; (h) Miller, W. D.; Fray, A. H.; Quatroche, J. T.; Sturgill, C. D. Org. Process Res. Dev. 2007, 11, 359; (i) Billingsley, K. L.; Buchwald, S. L. J. Org. Chem. 2008, 73, 5589. Mechanism: (j) Lam, K.C.; Marder, T.B.; Lin, Z. Organometallics 2010, 29, 1849; (k) Dang, L.; Lin, Z.; Marder, T.B. Chem. Commun. 2009, 3987. For borylation with aminoborane reagent, see: (1) Euzenat, L.; Horhant, D.; Ribourdouille, Y.; Duriez, C.; Alcaraz, G.; Vaultier, M. Chem. Commun. 2003, 2280. For a related Cu-catalyzed borylation, see: (m) Zhu, W.; Ma, D. Org. Lett. 2006, 8, 261 . For a related Ni-catalyzed borylation with in situ prepared 5,5-dimethyl-1,3,2-dioxaborinane, see: (n) Rosen, B. M.; Huang, C.; Percec, V. Org. Lett. 2008, 10, 2597; (o) Moldoveanu, C.; Wilson, D. A.; Wilson, C. J.; Corcoran, P.; Rosen, B. M.; Percec, V. Org. Lett. 2009, 11, 4974; (p) Wilson, D. A.; Wilson, C. J.; Moldoveanu, C.; Resmerita, A.M.; Corcoran, P.; Hoang, L. M.; Rosen, B. M.; Percec, V. J. Am. Chem. Soc. 2010, 1800; (q) Morgan, A. B.; Jurs, J. L.; Tour, J. M. J. Appl. Polym. Sci. 2000, 76, 1257. Arene C-H borylation has been recently reviewed: (r) Mkhalid, I. A. I.; Barnard, J. H.; Marder, T. B.; Murphy, J. M.; Hartwig, J. F. Chem. Rev. 2010, 110, 890.

(2) Murata, M.; Oda, T.; Watanabe, S.; Masuda, Y. Synthesis 2007, 351 .

(3) PraveenGanesh, N.; Chavant, P. Y. Eur. J. Org. Chem. 2008, 4690.

(4) (a) Chavant, P. Y.; PraveenGanesh, N. In e-EROS Encyclopaedia of Reagents for Organic Synthesis, John Wiley \& Sons, Ltd., 2008. DOI: 10.1002/047084289X.rn01065; (b) Woods, W. G.; Strong, P. L. J. Am. Chem. Soc. 1966, 88, 4667; (c) Fish, R. H.; Newsom, H. C. FR 1536699, US 19670427, 1968; (d) Kono, H.; Ito, K.; Nagai, Y. Chem. Lett. 1975, 1095; (e) Männig, D.; Nöth, H. Angew. Chem.
Int. Ed. 1985, 24, 878; (f) PraveenGanesh, N.; d'Hondt, S.; Chavant, P. Y. J. Org. Chem. 2007, 72, 4510.

(5) Wilkinson, H. S.; Tanoury, G. J.; Wald, S. A.; Senanayake, C. H. Org. Process Res. Dev. 2002, 6, 146.

(6) (a) Couturier, M.; Tucker, J. L.; Andresen, B. M.; Dube, P.; Negri, J. T. Org. Lett. 2001, 3, 465; (b) Couturier, M.; Tucker, J. L.; Andresen, B. M.; Dube, P.; Brenek, S. J.; Negri, J. T. Tetrahedron Lett. 2001, 42, 2285.

(7) Preparation of MPBH: under a well-ventilated fume hood ( $\mathrm{H}_{2}$ evolution), a 100-mL flask equipped with a Claisen distillation head was charged with $320 \mathrm{mg}$ of $10 \% \mathrm{Pd} / \mathrm{C}$ and anhydrous dioxane $(10 \mathrm{~mL})$. The flask was flushed with nitrogen and freshly distilled 2-methyl-2,4pentanediol $(50 \mathrm{mmol}, 6.4 \mathrm{~g})$ in dioxane $(10 \mathrm{~mL})$ was added. A solution of $N, N$-diethylaniline-borane $(60 \mathrm{mmol}$, $9.78 \mathrm{~g})$ in dioxane $(10 \mathrm{~mL})$ was added at $20^{\circ} \mathrm{C}$ over $30 \mathrm{~min}$. The reaction mixture was stirred for an additional $30 \mathrm{~min}$ at $20^{\circ} \mathrm{C}$. Low pressure distillation gave neat MPBH $(4.8 \mathrm{~g}$, $75 \%$, b.p. $45{ }^{\circ} \mathrm{C} / 35$ Torr).

(8) Our attempts to use $N, N$-diethylaniline as the base in subsequent borylations failed.

(9) [1,1'-biphenyl]-2-yl-dicyclohexyl-phosphine; Wolfe, J. P.; Singer, R. A.; Yang, B. H.; Buchwald, S. L. J. Am. Chem. Soc. 1999, 121, 9550 .

(10) Typical procedure for the borylation: an oven-dried Schlenk vessel (or a 10-mL microwaves vial) was charged with $\mathrm{Pd}_{2}(\mathrm{dba})_{3}(2.3 \mathrm{mg}, 2.5 \mu \mathrm{mol}$, described as $0.5 \%$ in Table 1) and CyJohnPhos (3.5 mg, $10 \mu \mathrm{mol}$, always 2 eq./Pd) and placed under an atmosphere of Argon. Anhydrous dioxane $(0.6 \mathrm{~mL})$, the aryl halide $(0.5 \mathrm{mmol})$, triethylamine (152 mg, $1.5 \mathrm{mmol}$ ) and MPBH (96 mg, 0.75 mmol) were introduced (solid aryl halides were added along with the other solid reagents). The reaction mixture was then heated at the indicated temperature until the aryl halide has been completely consumed as determined by gas chromatography. The reaction was allowed to cool to room temperature, and filtered through a thin pad of Celite (eluent ether). The eluate was concentrated and the crude material purified via flash chromatography on silica gel. Products $\mathbf{2 a -} \mathbf{j}^{2,3}, \mathbf{3 g}^{\mathrm{lb}}$ and $\mathbf{3 h}{ }^{1 \mathrm{i}}$ have been previously described. 4,4,6-trimethyl-2-naphthalen-2-yl-[1,3,2]dioxaborinane $\mathbf{2 g}$ : ${ }^{1} \mathrm{H}$ NMR $\left(400 \mathrm{MHz}, \mathrm{CDCl}_{3}\right) \delta \mathrm{ppm} 8.34(\mathrm{~s}, 1 \mathrm{H}), 7.89-7.85$ $(\mathrm{m}, 2 \mathrm{H}), 7.82-7.76(\mathrm{~m}, 2 \mathrm{H}), 7.48-7.40(\mathrm{~m}, 2 \mathrm{H}), 4.38(\mathrm{dqd}$, $J=11.8,6.2,2.9 \mathrm{~Hz}, 1 \mathrm{H}), 1.87(\mathrm{dd}, J=13.7,2.9 \mathrm{~Hz}, 1 \mathrm{H})$, $1.62(\mathrm{dd}, J=13.7,11.8 \mathrm{~Hz}, 1 \mathrm{H}), 1.41(\mathrm{~s}, 3 \mathrm{H}), 1.39(\mathrm{~s}, 3 \mathrm{H})$ $1.38(\mathrm{~d}, J=6.2 \mathrm{~Hz}, 3 \mathrm{H}) ;{ }^{13} \mathrm{C} \mathrm{NMR}\left(75.5 \mathrm{MHz}, C D C l_{3}\right) \delta$ ppm 134.8 (x2), 132.99, 130.14, 128.68, 127.67, 129.5 (very broad, C-B), 126.62, 126.39, 125.44, 71.16, 65.14, $46.15,31.38,28.27,23.30 ;{ }^{11} \mathrm{~B}$ NMR (128 $\mathrm{MHz}^{\left.-\mathrm{CDCl}_{3}\right) \delta}$ ppm 27.10; IR (neat, $\mathrm{cm}^{-1}$ ): 3053, 2972, 2932, 2911, 1630, 1598, 1503, 861, 823, 765, 746, 685; MS (EI, 70eV) 255 (26), 254 (100), 253 (28).

(11) (a) Baudoin, O.; Decor, A.; Cesario, M.; Gueritte, F. Synlett 2003, 2009; (b) Joncour, A.; Decor, A.; Thoret, S.; Chiaroni, A.; Baudoin, O. Angew. Chem. Int. Ed. 2006, 45, 4149; (c) Poriel, C.; Lachia, M.; Wilson, C.; Davies, J. R.; Moody, C. J. J. Org. Chem. 2007, 72, 2978.

(12) (a) Combs, A.; Zhu, W.; Crawley, M.; Glass, B.; Polam, P.; Sparks, R. B.; Modi, D.; Takvorian, A.; McLaughlin, E.; Yue,E.; Wasserman, Z.; Bower, M.; Wei, M.; Rupar, M.; Ala, P. J.; Reid, B. M.; Ellis, D.; Gonneville, L.; Emm, T.; Taylor, N.; Yeleswaram, S.; Li, Y.; Wynn, R.; Burn, T. C.; Hollis, G.; Liu, P. C. C.; Metcalf, B. J. Med. Chem. 2006, 49, 3774; (b) Mentzel, U. V.; Tanner, D.; Tonder, J. E. J. Org. Chem. 2006, 71, 5807.

(13) For related room-temperature Suzuki couplings, see: (a) Wolfe, J. P.; Tomori, H Sadighi, J. P.; Yin, J.; Buchwald, S. L. J. Org. Chem. 2000, 65, 1158; (b) Billingsley, K. L.; 
Barder, T. E.; Buchwald, S. L. Angew. Chem. Int. Ed. 2007, 46, 5359.

(14) Very recently, Murata et al. borylated aryl chlorides with both PinBH and MPBH in the presence of excess $\mathrm{Bu}_{4} \mathrm{NI}$ : Murata, M.; Sambommatsu, T.; Oda, T.; Watanabe, S.; Masuda, Y. Heterocycles 2010, 80, 213.

(15) (a) Wakim, S.; Bouchard, J.; Simard, M.; Drolet, N.; Tao, Y.; Leclerc, M. Chem. Mater. 2004, 16, 4386; (b) Ahmed, V.; Liu, Y.; Silvestro, C.; Taylor, S. D. Bioorg. Med. Chem. 2006, 14, 8564; (c) Tam, V. K.; Liu, Q.; Tor, Y. Chem. Commun. 2006, 2684.

(16) See Supporting Information for a detailed table of these experiments.
(17) So, C. M.; Lau, C. P.; Kwong, F. Y. Org. Lett. 2007, 9, 2795.

(18) (a) Altemoeller, M.; Podlech, J.; Fenske, D. Eur. J. Org. Chem. 2006, 1678; (b) Suzuki, A. Proc. Jpn. Acad. 2004, $80,359$.

(19) The disproportionation of dialkoxyboranes yields trialkoxyboranes and $\mathrm{BH}_{3}$. See: (a) Rose, S. H.; Shore, S. G. Inorg. Chem. 1962, 1, 744; (b) Pasto, D. J.; Balasubramaniyan, V.; Wojtkowski, P.W. Inorg. Chem. 1969, 8, 594. The reaction is influenced by phosphines and/or metal species: Hadebe, S.W.; Robinson, R. S. Eur. J. Org. Chem. 2006, 4898 and references therein. 


\section{Efficient borylation of reactive aryl halides with MPBH (4,4,6-trimethyl- 1,3,2-dioxaborinane).}

DOI:

Nageswaran PraveenGanesh, Emilien Demory, Christine Gamon, Véronique Blandin, Pierre Y. Chavant*

Published in

SYNLETT 2010, No. x, pp 0001-0004

Advanced online publication: $\mathrm{xx} . \mathrm{xx} .2010$

DOI: 10.1055/s-0030-xxxxx; Art ID: D10810ST

(C) Georg Thieme Verlag Stuttgart · New York

This article describes our study on borylation of aryl halides and triflates with 4,4,6-trimethyl-1,3,2-dioxaborinane (MPBH), a stable and easily available reagent. The combination of MPBH and Buchwald's palladium catalyst provides a simple, fast, cost-effective borylation of electron-rich, reactive aryl iodides, bromides, and triflates, to produce stable, easily purified boronic esters.

In the course of this work we found that the desired borylation can be hampered by a concurrent hydrodehalogenation of the substrate. We studied the influence of various parameters on the extent of this side-reaction in the case of 2bromonaphtalene as the model. The results of this study are summarized in the table below.

\section{Standard procedure for the borylation of 2-bromonaphtalene}

An oven-dried Schlenk vessel (or a $10-\mathrm{mL}$ microwaves vial) was charged with 2-bromonaphtalene (0.5 mmol), $\mathrm{Pd}_{2}(\mathrm{dba})_{3}(2.3 \mathrm{mg}, 0.5 \%)$ and CyJohnPhos $(3.5 \mathrm{mg}, 2 \%)$ and placed under an atmosphere of Argon. Anhydrous toluene $(1 \mathrm{~mL})$, triethylamine $(152 \mathrm{mg}, 1.5 \mathrm{mmol}), \mathrm{MPBH}(96 \mathrm{mg}, 0.75 \mathrm{mmol})$ and tetradecane (as an internal standard) were introduced with a syringe. The reaction mixture was then heated at the indicated temperature for the indicated time. When microwaves were used the reaction was hydrolysed after this time and analyzed by GC (relative response factors of the components were determined from mixtures of pure samples). Reactions performed in an oil bath at atmospheric pressure were monitored by repeated analysis of small aliquots. 


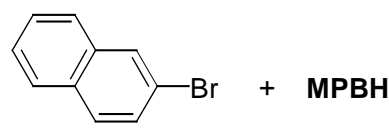

$\mathrm{ArBr}$

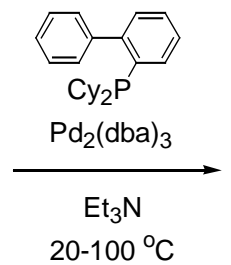

toluene

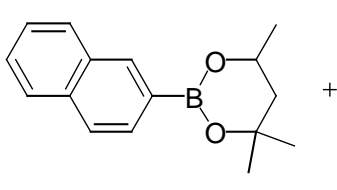

ArMPB

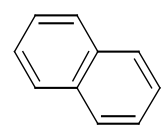

ArH

Scheme: Borylation of 2-bromonaphtalene

\begin{tabular}{|c|c|c|c|c|c|}
\hline Entry & temp. & time & deviation from standard conditions & conversion & Ar-MPB:Ar-H \\
\hline 18 & $40^{\circ} \mathrm{C}$ (thermal) & $16 \mathrm{hrs}$ & 3 eq. $\mathrm{MPBH}$ & 99 & $38: 62$ \\
\hline 19 & $80^{\circ} \mathrm{C}$ (thermal) & $6 \mathrm{hrs}$ & - & 100 & $37: 63$ \\
\hline 20 & $110^{\circ} \mathrm{C}$ (thermal) & $20 \mathrm{~min}$ & 3 eq. $\mathrm{MPBH}$ & 100 & $35: 65$ \\
\hline 21 & $110^{\circ} \mathrm{C}$ (thermal) & $30 \mathrm{~min}$ & - & 49 & 39:61 \\
\hline 22 & $110^{\circ} \mathrm{C}(\mu \mathrm{W})$ & $30 \mathrm{~min}$ & - & 55 & $42: 58$ \\
\hline 23 & $110^{\circ} \mathrm{C}$ (thermal) & $16 \mathrm{hrs}$ & - & 100 & $44: 56$ \\
\hline 24 & $150^{\circ} \mathrm{C}(\mu \mathrm{W})$ & $5 \mathrm{~min}$ & - & 100 & $45-50: 50-55^{1}$ \\
\hline 25 & $180^{\circ} \mathrm{C}(\mu \mathrm{W})$ & $5 \mathrm{~min}$ & - & 100 & $50: 50$ \\
\hline 26 & $150^{\circ} \mathrm{C}(\mu \mathrm{W})$ & $5 \mathrm{hrs}$ & $0,1 \mathrm{~mol} \% \mathrm{Pd}, 0.2 \mathrm{~mol} \%$ ligand & 82 & $49: 51$ \\
\hline 27 & $150^{\circ} \mathrm{C}(\mu \mathrm{W})$ & $30 \mathrm{~min}$ & 1 eq $\mathrm{Et}_{3} \mathrm{~N}$ & 78 & 49:51 \\
\hline 28 & $150^{\circ} \mathrm{C}(\mu \mathrm{W})$ & $5 \mathrm{~min}$ & 1.2 eq. MPBH & 87 & $47: 53$ \\
\hline 29 & $150^{\circ} \mathrm{C}(\mu \mathrm{W})$ & $15 \mathrm{~min}$ & 1.2 eq. $\mathrm{MPBH}$ & 98 & $44: 56$ \\
\hline 30 & $150^{\circ} \mathrm{C}(\mu \mathrm{W})$ & $30 \mathrm{~min}$ & $\begin{array}{l}1.2 \text { eq. } \mathrm{MPBH}, \\
\text { dioxane }\end{array}$ & 95 & $43: 57$ \\
\hline 31 & $150^{\circ} \mathrm{C}(\mu \mathrm{W})$ & $30 \mathrm{~min}$ & $\mathrm{Pd}$ as $\mathrm{Pd}(\mathrm{AcO})_{2}$ & 100 & $48: 52$ \\
\hline 32 & $150^{\circ} \mathrm{C}(\mu \mathrm{W})$ & $30 \mathrm{~min}$ & $\begin{array}{c}2 \text { mol\% } N \text {-(dicyclohexylphosphino) } \\
\text {-2-(2'-tolyl)indole }\end{array}$ & 84 & $46: 54$ \\
\hline 33 & $150^{\circ} \mathrm{C}(\mu \mathrm{W})$ & $30 \mathrm{~min}$ & 1mol\% DPEphos & 55 & $43: 57$ \\
\hline 34 & $150^{\circ} \mathrm{C}(\mu \mathrm{W})$ & $60 \mathrm{~min}$ & $1 \mathrm{~mol} \% \mathrm{dppf}$ & 100 & 39:61 \\
\hline 35 & $150^{\circ} \mathrm{C}(\mu \mathrm{W})$ & $30 \mathrm{~min}$ & $2 \mathrm{~mol} \%$ TPP & 100 & $33: 67$ \\
\hline 36 & $110^{\circ} \mathrm{C}$ (thermal) & $110 \mathrm{~min}$ & 3 eq. MPBH, 4 mol\% TPP, dioxane & 100 & $21: 79$ \\
\hline 37 & $150^{\circ} \mathrm{C}(\mu \mathrm{W})$ & $30 \mathrm{~min}$ & $1 \mathrm{~mol} \% \mathrm{dtbpy}^{2}$ & 61 & $13: 87$ \\
\hline 38 & $150^{\circ} \mathrm{C}(\mu \mathrm{W})$ & $30 \mathrm{~min}$ & $\mathrm{Pd} / \mathrm{C}$, no ligand & 65 & 3:97 \\
\hline
\end{tabular}

${ }^{1}$ run repeated thrice.

${ }^{2}$ 4,4'-di-tert-butyl-2,2' -dipyridyl 


\section{Typical microwaves experiment: heating profiles}

The profiles below (entry 31) are typical. This reaction was performed on a $0.5 \mathrm{mmol}$ scale in a $10 \mathrm{~mL}$ vessel; solvent was toluene.

CEM DISCOVER apparatus,

Method Parameters

Prestirring(mm:ss): 00:15

Type:Dynamic

$\begin{array}{ccc}\text { Stage } & \operatorname{Temp}(\mathrm{C}) & \text { Time(mm:ss) } \\ 1 & 150 & 30: 00\end{array}$

Pressure(BAR)

Power(W) PowerMAX

17,0

300

Stirring

Graphs
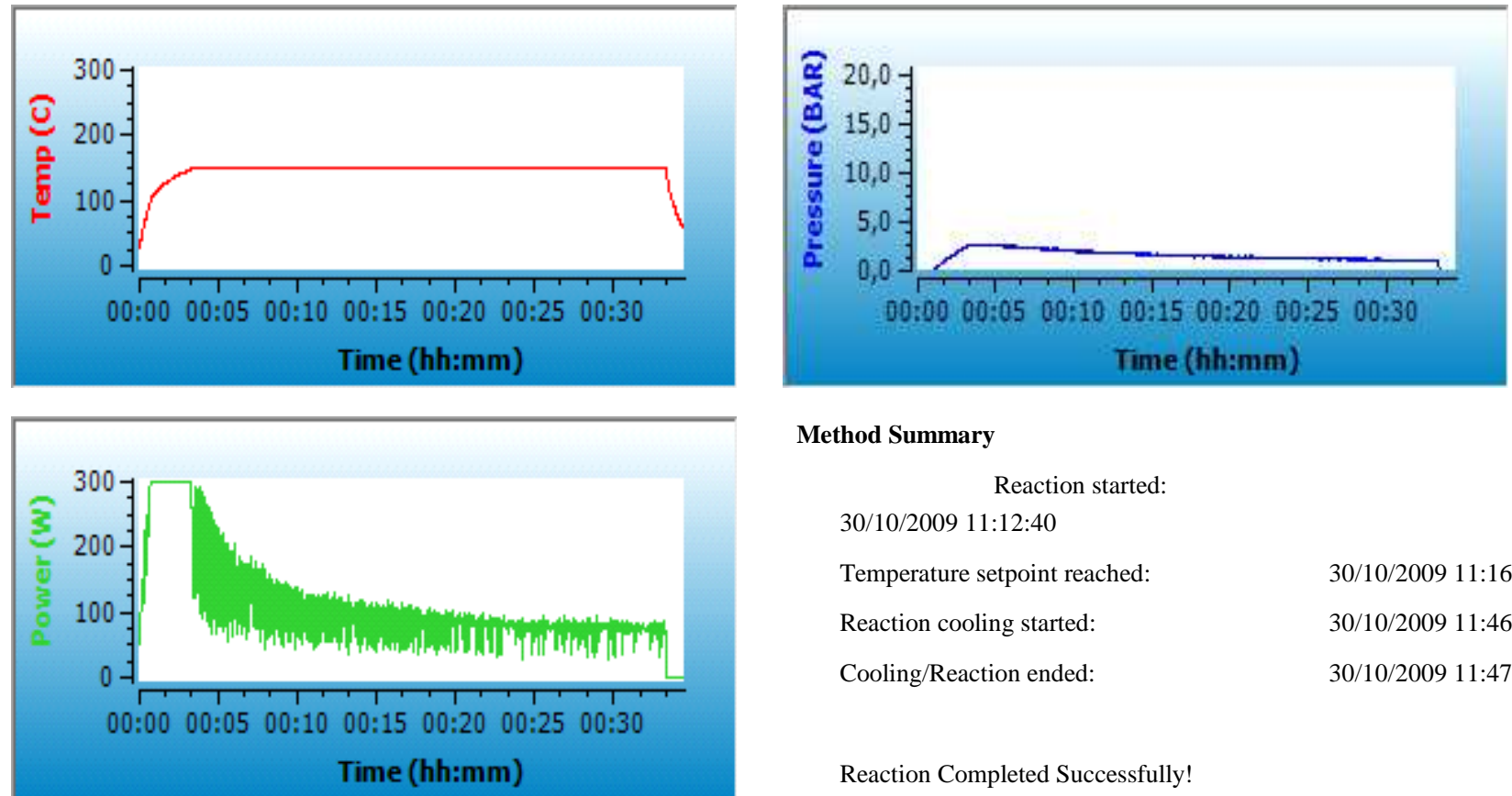

Method Summary

Reaction started:

30/10/2009 11:12:40

Temperature setpoint reached:

30/10/2009 11:16:00

Reaction cooling started:

30/10/2009 11:46:01

Cooling/Reaction ended:

30/10/2009 11:47:07

Reaction Completed Successfully!

Maximum temperature:

Maximum pressure:

Time to obtain setpoint:

Time at setpoint:

Time cooling:
$151 \mathrm{C}$

3 BAR

03:20 mm:ss

30:01 mm:ss

01:06 mm:ss 